\title{
Chromogranin A as a biomarker for prostate cancer: is it actually relevant for clinical practice?
}

\author{
Marialuisa Appetecchia*,1, Rosa Lauretta ${ }^{1}$, Isabella Sperduti ${ }^{2}$ \& Michele Gallucci $^{3}$ \\ ${ }^{1}$ Endocrinology Unit, Regina Elena National Cancer Institute, Rome, Italy \\ ${ }^{2}$ Biostatistical Unit, Regina Elena National Cancer Institute, Rome, Italy \\ ${ }^{3}$ Urology Unit, Regina Elena National Cancer Institute, Rome, Italy \\ *Author for correspondence: marialuisa.appetecchia@ifo.gov.it
}

"We believe that the CgA assessment may maintain some prognostic utility in certain cases, such as in combination with NSE assessment or in patients treated with certain specific therapies (e.g., abiraterone). On the other hand, the role of $\mathrm{CgA}$ in these patients needs further study, with the aim of improving the knowledge on the molecular basis of the disease."

First draft submitted: 11 January 2018; Accepted for publication: 16 January 2018; Published online: 1 May 2018

The diagnosis of prostate cancer is often based on the evaluation of biomarkers, in particular the prostate specific antigen (PSA), which may allow the early detection of the disease and the immediate establishment of curative therapy [1]. However, PSA has very limited specificity and therefore unnecessary biopsies and misdiagnoses are frequent in clinical practice [1,2]. There is, therefore, a strong need for other prognostic biomarkers that are also able to distinguish between inactive and aggressive disease forms $[1,3,4]$. For example, current research lines are exploring the use of urinary biomarkers and genomic tools [1,4].

Among the various biomarkers tested to date in clinical research, chromogranin $\mathrm{A}(\mathrm{CgA})$, a member of the neuroendocrine secretory protein family, is synthesized by neuroendocrine cells in the prostate and is an established marker of neuroendocrine differentiation [2]. In recent years, the prognostic capacity of $\operatorname{CgA}$ as a biomarker of prostate cancer has been extensively evaluated [2,3,5-10]. However, to date the results remain widely controversial $[2,3]$.

In the last few months, three important studies on CgA as a biomarker have been published [5-7]. Heck et al. evaluated the impact of elevated neuroendocrine serum markers, namely CgA and neuron-specific enolase (NSE), on the treatment outcome in a small series $(n=45)$ of patients with castration-resistant metastatic prostate cancer (mCRPC) who were receiving abiraterone [5]. Patients were stratified into low, intermediate, or high-risk groups based on the elevation of none, one or both neuroendocrine markers, respectively. Patients in the low-risk group showed prolonged overall survival (OS) compared with those in the other groups (not achieved vs 15.3 vs 6.6 months; $\mathrm{p}<0.001)$. Similar results were reported for clinical progression or radiographic progression-free survival (PFS), but no correlation with the PSA response was observed. In another study, Niedworok et al. tested the association between serum CgA levels and long-term disease-specific survival [6]. CgA levels were significantly elevated in patients with advanced disease, compared with those with clinically localized carcinoma, both in serum and plasma samples ( $45 \mathrm{vs} 23 \mathrm{ng} / \mathrm{ml}, \mathrm{p}<0.001$ and $41 \mathrm{vs} 22 \mathrm{ng} / \mathrm{ml}, \mathrm{p}=0.002$ ). In agreement, high levels of CgA correlated with poor DSS; however, in clinically localized disease, CgA levels alone were not prognostic. Finally, Fan et al. evaluated the prognostic utility of serum CgA and NSE variations in the first 3 months of treatment with abiraterone in a limited series $(n=40)$ of patients with metastatic castration-resistant prostate cancer [7]. Overall, the combination of CgA and NSE ( $\geq 1$ positive marker vs both negative markers) and the combination of CgA and NSE elevation during the first 3 months of treatment were predictors of OS and radiographic PFS.

These results are overall in line with those reported by our group's extensive experience published in 2010 [9]. In more detail, we analyzed the incidence of preoperative serum CgA levels in 486 patients with nonmetastatic prostate cancer treated in our center between 2003 and 2006. Overall, the CgA was elevated (>90 ng/ml) in 114 subjects (23.5\%). Worthy of note, the prevalence of elevated CgA increased with the Gleason score: it was $25.5 \%$ in subjects with a Gleason score $<7$ and $31.4 \%$ in patients with a Gleason score $>7$ (18.5\% of patients with Gleason

Future $\because$ Medicine 
score $=7$ showed elevated $\mathrm{CgA}$ ). On this basis, we were able to conclude that the circulating preoperative $\mathrm{CgA}$ could supplement the PSA in the selection of more aggressive cases of prostate cancer, particularly in the presence of a higher Gleason score [9]. However, it should be noted that all of those studies, including ours, were conducted in small series or patients and/or had a short follow-up.

Apparently, according to the above-mentioned experiences including ours, CgA levels could have a potential role as a survival biomarker and tumor aggression in prostate cancer, when considered in combination with other biomarkers such as NSE. But are these results still valid over a longer follow-up?

To our knowledge, no long-term study has formally evaluated the correlation between CgA levels and survival. Therefore, with an exclusively exploratory intent, in October 2017 we examined the clinical data of the patients enrolled in the study reported above by our group [9] and that have been followed at our center, in order to evaluate their survival at a median follow-up of 50 months (range: 1-119). These results are shown below and will be the subject of a more complete analysis. In total, we were able to evaluate 274 patients (mean age: 64 years at surgery; 151 with a Gleason score $<7)$. The majority of patients were classified as intermediate risk $(\mathrm{n}=159,60 \%)$ [9]. Patients at low and high risk were $12(5 \%)$ and $95(35 \%)$, respectively. The majority of patients $(\mathrm{n}=159,60 \%)$ were followed according to a 'watchful waiting' approach at the time of the analysis, while chemotherapy/radiotherapy was established in 90 subjects (33\%) and hormone therapy in 65 (24\%). In total, 211 patients (77\%) had normal values $(<90 \mathrm{mg} / \mathrm{ml})$ of serum CgA levels at diagnosis, while high levels of CgA were reported in 63 subjects (23\%). No difference between these two subpopulations was observed in terms of median age, stage of disease, PSA at diagnosis, Gleason score, risk class or therapeutic decision. A preliminary Kaplan-Meier analysis showed that the long-term PFS trend was perfectly comparable in patients with CgA elevated at diagnosis and in those with normal values (Supplementary Figure 1). Moreover, the 7-year PFS rate was 69\% in patients with normal CgA and 65\% in those with high values $(\mathrm{p}=0.63)$.

Overall, these findings may suggest that the potential prognostic capacity of baseline $\mathrm{CgA}$ in prostate cancer may be lost during long-term follow-up, as seen in many cases in clinical practice, given the increased survival achieved with current treatments. On the basis of the available evidence, can this CgA assessment have real relevance in everyday practice, also from a cost-effectiveness perspective? We believe that the CgA assessment may maintain some prognostic utility in certain cases, such as in combination with NSE assessment or in patients treated with certain specific therapies (e.g., abiraterone). On the other hand, the role of CgA in these patients needs further study, with the aim of improving the knowledge on the molecular basis of the disease.

Supplementary data

To view the supplementary data that accompany this paper please visit the journal website at: www.futuremedicine.com/doi/full/ $10.2217 /$ fon-2018-0025

\section{Acknowledgements}

The authors would like to thank L Giacomelli (University of Genoa, Genoa, Italy, an Regina Elena Cancer Institute, Rome, Italy) for useful discussion.

\section{Financial \& competing interests disclosure}

The authors have no relevant affiliations or financial involvement with any organization or entity with a financial interest in or financial conflict with the subject matter or materials discussed in the manuscript. This includes employment, consultancies, honoraria, stock ownership or options, expert testimony, grants or patents received or pending, or royalties.

No writing assistance was utilized in the production of this manuscript.

\section{References}

1. Cucchiara V, Cooperberg MR, Dall'Era M et al. Genomic markers in prostate cancer decision making. Eur. Urol. 73(4), 572-582 (2018).

2. Esfahani M, Ataei N, Panjehpour M. Biomarkers for evaluation of prostate cancer prognosis. Asian Pac. J. Cancer Prev. 16(7), 2601- 2611 (2015).

3. Grigore AD, Ben-Jacob E, Farach-Carson MC. Prostate cancer and neuroendocrine differentiation: more neuronal, less endocrine? Front. Oncol. 5, 37 (2015).

4. Wu D, Ni J, Beretov J et al. Urinary biomarkers in prostate cancer detection and monitoring progression. Crit. Rev. Oncol. Hematol. 118, $15-26$ (2017). 
5. Heck MM, Thaler MA, Schmid SC et al. Chromogranin A and neurone-specific enolase serum levels as predictors of treatment outcome in patients with metastatic castration-resistant prostate cancer undergoing abiraterone therapy. BJU Int. 119(1), 30-37 (2017).

6. Niedworok $\mathrm{C}$, Tschirdewahn $\mathrm{S}$, Reis $\mathrm{H}$ et al. Serum chromogranin $\mathrm{A}$ as a complementary marker for the prediction of prostate cancer-specific survival. Pathol. Oncol Res. 23(3), 643-650 (2017).

7. Fan L, Wang Y, Chi C et al. Chromogranin A and neurone-specific enolase variations during the first 3 months of abiraterone therapy predict outcomes in patients with metastatic castration-resistant prostate cancer. BJU Int. 120, 226-232 (2017).

8. Mitsui Y, Arichi N, Hiraki M et al. Tissue chromogranin A expression during prostate cancer progression: prediction of chemosensitivity. Urol. J. 12(3), 2165-2172 (2015).

9. Appetecchia M, Meçule A, Pasimeni G et al. Incidence of high chromogranin A serum levels in patients with non metastatic prostateadenocarcinoma. J. Exp. Clin. Cancer Res. 29, 166 (2010).

10. De Nunzio C, Albisinni S, Presicce F et al. Serum levels of chromogranin A are not predictive of high-grade, poorly differentiated prostate cancer: results from an Italian biopsy cohort. Urol. Oncol. 32(2), 80-84 (2014). 
